\title{
On the topological stable rank of non-selfadjoint operator algebras
}

\author{
K. R. Davidson - R. H. Levene - L. W. Marcoux • \\ H. Radjavi
}

Published online: 1 April 2008

(C) Springer-Verlag 2008

\section{Erratum to: Math. Ann.}

\section{DOI 10.1007/s00208-007-0180-5}

There is an error in our paper in the proof of Theorem 2.11. The purpose of this note is to make the necessary corrections. We thank Prof. You Qing Ji for pointing out the mistake, which occurs in the last line of the proof: At this point, we can apply the second half of the proof of Theorem 2.1. In fact, some non-trivial modifications of that proof are required.

Theorem 2.11 Suppose that $\mathcal{N}$ is a nest ordered like $\omega$, all of whose atoms are finite dimensional. Let $A_{n}, n \geq 1$, denote the atoms of $\mathcal{N}$, and let $r_{n}=\operatorname{dim} A_{n}$ for $n \geq 1$. Set $R(k)=\max _{1 \leq i \leq k} r_{i}$ for $k \geq 1$. Suppose that there exists $a \gamma>0$ and an integer $J>0$ such that

$$
R((k+1) J) \geq(1+\gamma) R(k J) \text { for all } k \geq 1
$$

The online version of the original article can be found under doi:10.1007/s00208-007-0180-5.

K. R. Davidson · L. W. Marcoux $(\varangle) \cdot$ H. Radjavi

Department of Pure Mathematics, University of Waterloo, Waterloo, ON, Canada N2L 3G1

e-mail: LWMarcoux@uwaterloo.ca

K. R. Davidson

e-mail: krdavids@uwaterloo.ca

H. Radjavi

e-mail: hradjavi@uwaterloo.ca

R. H. Levene

Department of Pure Mathematics, Queen's University Belfast,

Belfast BT7 1NN, Northern Ireland, UK

e-mail: r.levene@qub.ac.uk 
Then

$$
\operatorname{ltsr}(\mathcal{T}(\mathcal{N}))=\infty \text { and } \operatorname{rtsr}(\mathcal{T}(\mathcal{N}))=2
$$

Proof The proof as published remains the same up to the last paragraph. We also define $Q_{k}=\sum_{i=1}^{k} F_{k}$.

We wish to multiply $A^{\prime}$ and $B^{\prime}$ on the right by invertible elements of $\mathcal{T}(\mathcal{N})$ to make the block diagonal equal to the identity and the first super-diagonal with respect to the block decomposition equal to 0 . To accomplish this, let $\Delta_{1}(T)=\sum_{k \geq 1} F_{k} T F_{k+1}$ for $T \in \mathcal{T}(\mathcal{N})$. Then define

$$
X=D_{a}^{-1} \exp \left(-\Delta_{1}\left(A^{\prime}\right) D_{a}^{-1}\right) \quad \text { and } \quad Y=D_{b}^{-1} \exp \left(-\Delta_{1}\left(B^{\prime}\right) D_{b}^{-1}\right) .
$$

Then $A^{\prime} X=I+X_{0}$ and $B=I+Y_{0}$ where $X_{0}$ and $Y_{0}$ lie in the ideal

$$
\mathcal{T}_{0}^{\prime}(\mathcal{N})^{2}=\left\{T \in \mathcal{T}(\mathcal{N}): \sum_{k \geq 1} F_{k} T F_{k}=0=\sum_{k \geq 1} F_{k} T F_{k+1}\right\} .
$$

So we may write $X_{0}=\sum_{k \geq 3} Q_{k-2} X_{0} F_{k}$ and $Y_{0}=\sum_{k \geq 3} Q_{k-2} Y_{0} F_{k}$.

Arguing as in the proof of Theorem 2.1, we see that

$$
\operatorname{rank}\left(X_{0} F_{k}\right)+\operatorname{rank}\left(Y_{0} F_{k}\right) \leq \frac{1}{2} \operatorname{rank} F_{k-1} .
$$

Thus we can choose projections $P_{k} \leq F_{k}$ with rank $P_{k} \leq \frac{1}{2}$ rank $F_{k-1}$ so that $X_{0} F_{k}=$ $X_{0} P_{k}$ and $Y_{0} F_{k}=Y_{0} P_{k}$. The problem with a trivial modification of the argument is that we can no longer assert that $P_{k}$ and the partial isometries constructed in the proof of Theorem 2.1 belong to the nest algebra. However, because we have made some extra room to maneuver, we can construct partial isometries $U_{k}$ mapping $P_{k} \mathfrak{H}$ into $P_{k-1}^{\perp} F_{k-1} \mathfrak{H}$; and these operators evidently lie in the nest algebra. Set $U=\sum_{k \geq 3} U_{k}$. Moreover

$$
X_{0} U^{*}=\sum_{k \geq 3} Q_{k-2} X_{0} P_{k} \sum_{j \geq 3} P_{j} U_{j}^{*}=\sum_{k \geq 3} Q_{k-2}\left(X_{0} P_{k} U_{k}^{*}\right) F_{k-1} .
$$

So this operator also lies in $\mathcal{T}(\mathcal{N})$. Similarly $Y_{0} U^{*}$ belongs to the nest algebra. Moreover the range of $U$ is orthogonal to the range of $P=\sum_{k \geq 3} P_{k}$, and therefore $X_{0} U=Y_{0} U=0$.

Now let $A^{\prime \prime}=A^{\prime}+\delta X_{0} U^{*} X^{-1}$ and $B^{\prime \prime}=B^{\prime}-\delta Y_{0} U^{*} Y^{-1}$, where $\delta>0$ is chosen to be sufficiently small so that $\left\|A-A^{\prime \prime}\right\|<\varepsilon$ and $\left\|B-B^{\prime \prime}\right\|<\varepsilon$. Observe that

$$
\begin{aligned}
& A^{\prime \prime} X\left(I-\delta^{-1} U\right)+B^{\prime \prime} Y\left(I+\delta^{-1} U\right) \\
& \quad=\left(I+X_{0}+\delta X_{0} U^{*}\right)\left(I-\delta^{-1} U\right)+\left(I+Y_{0}-\delta Y_{0} U^{*}\right)\left(I+\delta^{-1} U\right) \\
& =\left(I+X_{0}+\delta X_{0} U^{*}-\delta^{-1} U-X_{0}\right)+\left(I+Y_{0}-\delta Y_{0} U^{*}+\delta^{-1} U-Y_{0}\right) \\
& =2 I+\delta\left(X_{0}-Y_{0}\right) U^{*} .
\end{aligned}
$$

With $\delta$ sufficiently small, this is invertible, and hence $\left[A^{\prime \prime} B^{\prime \prime}\right]$ is right invertible.

Therefore $\operatorname{rtsr}(\mathcal{T}(\mathcal{N}))=2$. 